\title{
Membrane Attachment Is Key to Protecting Transducin GTPase-Activating Complex from Intracellular Proteolysis in Photoreceptors
}

\author{
Sidney M. Gospe III, ${ }^{1 \star}$ Sheila A. Baker, ${ }^{1 \star}$ Christopher Kessler, ${ }^{2}$ Martha F. Brucato, ${ }^{1}$ Joan R. Winter, ${ }^{1}$ Marie E. Burns, ${ }^{2}$ \\ and Vadim Y. Arshavsky ${ }^{1}$ \\ ${ }^{1}$ Albert Eye Research Institute, Duke University, Durham, North Carolina 27710, and ${ }^{2}$ Center for Neuroscience and Department of Ophthalmology and \\ Vision Science, University of California, Davis, Davis, California 95618
}

\begin{abstract}
The members of the R7 regulator of G-protein signaling (RGS) protein subfamily are versatile regulators of G-protein signaling throughout the nervous system. Recent studies indicate that they are often found in complexes with membrane anchor proteins that serve as versatile modulators of their activity, intracellular targeting, and stability. One striking example is the interplay between the membrane anchor R9AP and the RGS9-1 - G $\beta 5$ GTPase-activating complex responsible for the rapid inactivation of the G-protein transducin in vertebrate photoreceptor cells during their recovery from light excitation. The amount of this complex in photoreceptors sets their temporal resolution and is precisely regulated by the expression level of R9AP, which serves to protect the RGS9-1 and G $\beta 5$ subunits from intracellular proteolysis. In this study, we investigated the mechanism by which R9AP performs its protective function in mouse rods and found that it is entirely confined to recruiting RGS9-1 $\cdot G \beta 5$ to cellular membranes. Furthermore, membrane attachment of RGS9-1 $\cdot G \beta 5$ is sufficient for its stable expression in rods even in the absence of R9AP. Our second finding is that RGS9-1 $\cdot G \beta 5$ possesses targeting information that specifies its exclusion from the outer segment and that this information is neutralized by association with R9AP to allow outer segment targeting. Finally, we demonstrate that the ability of R9AP - RGS9-1 $\cdot \mathrm{G} \beta 5$ to accelerate GTP hydrolysis on transducin is independent of its means of membrane attachment, since replacing the transmembrane domain of R9AP with a site for lipid modification did not impair the catalytic activity of this complex.
\end{abstract}

\section{Introduction}

G-proteins regulate numerous signaling pathways in eukaryotic cells. The strength and duration of a G-protein-mediated signal is determined by the balance between G-protein activation via GTP binding to the $\alpha$-subunit and inactivation achieved via GTP hydrolysis. The latter is typically regulated by GTPase-activating proteins of the regulator of G-protein signaling (RGS) family (Ross and Wilkie, 2000). Of particular significance to neuronal signaling are R7 RGS proteins, which exist as constitutive dimers with the atypical G-protein $\beta$-subunit $\mathrm{G} \beta 5$ and regulate multiple processes, including vision, motor control, reward behavior, and nociception (Anderson et al., 2009). Recent studies revealed that many aspects of R7 protein function are further modulated by

\footnotetext{
Received July 9, 2011; revised Aug. 14, 2011; accepted Aug. 19, 2011.

Author contributions:S.M.G., S.A.B., M.E.B., and V.Y.A. designed research;S.M.G., S.A.B., C.K., M.F.B., and J.R.W. performed research; S.M.G., S.A.B., C.K., M.F.B., J.R.W., M.E.B., and V.Y.A. analyzed data; S.M.G., S.A.B., M.E.B., and V.Y.A. wrote the paper.

This work was supported by NIH Grants EY12859 (V.Y.A.) and EY14047 (M.E.B.) and NIH Core Grant for Vision Research EY5722 (Duke University).

*S.M.G. and S.A.B. contributed equally to this work.

Correspondence should be addressed to Vadim Y. Arshavsky, Duke University Eye Center, 5012 AERI, 2351 Erwin

Road, Durham, NC 27710. E-mail: vadim.arshavsky@duke.edu.

S. A. Baker's present address: Department of Biochemistry, University of lowa, lowa City, IA 52242

DOI:10.1523/JNEUROSCI.3516-11.2011

Copyright $\odot 2011$ the authors $\quad 0270-6474 / 11 / 3114660-09 \$ 15.00 / 0$
}

membrane anchor proteins, R9AP and R7BP (Jayaraman et al., 2009).

A striking example of how an anchor protein influences R7 protein function is the R9AP $\cdot$ RGS9-1 - G $\beta 5$ GTPase-activating complex in vertebrate photoreceptors, which is responsible for rapid inactivation of transducin $\left(\mathrm{G} \alpha_{\mathrm{t}}\right)$ and normal recovery of the light response (for review, see Wensel, 2008; Martemyanov and Arshavsky, 2009). R9AP is a transmembrane protein that anchors RGS9-1 $-\mathrm{G} \beta 5$ to disc membranes of the outer segment compartment and performs three additional functions: enhancement of the ability of RGS9-1 $\cdot G \beta 5$ to inactivate $G \alpha_{t}$ by increasing the rate of GTP hydrolysis (Lishko et al., 2002; Hu et al., 2003; Baker et al., 2006); targeting of RGS9-1 - G $\beta 5$ to photoreceptor outer segments (Martemyanov et al., 2003); and protection of RGS9-1 - G $\beta 5$ from intracellular proteolysis, ultimately establishing the expression level of the entire complex (Keresztes et al., 2004; Krispel et al., 2006).

The protection of RGS9-1 $\cdot$ G $\beta 5$ by R9AP was inferred from two complementary studies. Photoreceptors of $R 9 A P^{-1-}$ mice were shown to lack RGS9-1 protein and to have greatly reduced levels of G $\beta 5$ protein, despite unaffected mRNA levels for both genes (Keresztes et al., 2004). Conversely, overexpression of R9AP (Krispel et al., 2006) [but not RGS9-1 or G $\beta 5$ (Chen et al., 2003; Krispel et al., 2006)] resulted in elevated expression of the entire R9AP - RGS9-1 $\cdot$ G $\beta 5$ complex. These observations suggest a posttranslational protection mechanism involving pro- 
tein-protein interactions. Indeed, an RGS9-1 mutant lacking the Disheveled/EGL-10/Pleckstrin (DEP) domain critical for R9AP binding was stably expressed in rods (Martemyanov et al., 2003), suggesting that the DEP domain destabilizes full-length RGS9-1, resulting in its rapid proteolysis (and consequently proteolysis of G $\beta 5$ ) unless bound and protected by R9AP. However, the mechanistic aspects of this protection remain poorly understood.

Here, we addressed this question by considering two plausible (and not mutually exclusive) mechanisms: (1) physical masking of labile RGS9-1 residues by R9AP and (2) R9AP-dependent sequestration of RGS9-1 - G $\beta 5$ from the proteolytic machinery of the rod. The latter could be accomplished either by confining RGS9-1 - G $\beta 5$ to outer segments (which lack lysosomes and proteasomes) or by simply recruiting the complex from the cytosol to cellular membranes. We distinguished between these possibilities by expressing an array of soluble or membrane-bound protein constructs in the rods of $R 9 A P^{-1-}$ mice. Subsequently, to further probe the functional properties of R9AP, we examined the electrophysiological behavior of rods expressing one of the constructs-R9AP attached to membranes via lipid modifications instead of a transmembrane domain.

\section{Materials and Methods}

Primary antibodies and DNA constructs. Rabbit polyclonal antibodies against $\mathrm{G} \beta 5$ and the $\mathrm{C}$ terminus of RGS9-1 were kind gifts from William Simonds (NIDDK-NIH, Bethesda, MD) and Theodore Wensel (Baylor College of Medicine, Houston, TX), respectively. The mouse monoclonal antibody 1D4 recognizing the $\mathrm{C}$ terminus of rhodopsin was a kind gift from Robert Molday (University of British Columbia, Vancouver, BC, Canada). Rabbit polyclonal antibodies against amino acids 144-223 of mouse R9AP are described by Keresztes et al. (2003), and sheep polyclonal antibodies against rat phosducin are described by Sokolov et al. (2004). Polyclonal anti-peptide antibody against bovine Rom-1 was generated in sheep by Elmira Biologicals using the peptide PAPGETPPEEKPPKECLPE conjugated to KLH. Affinity purification of antibody from sheep serum was achieved using the same peptide coupled to the Sulfolink Plus column (Pierce), following the manufacturer's instructions. Goat polyclonal anti-RGS9 (T-19) (sc-8143; Santa Cruz) and monoclonal mouse anti-FLAG M2 (Sigma-Aldrich) and anti- $\beta$-actin (sc-47778; Santa Cruz) antibodies were available commercially.

Plasmid DNA for pRho-DsRed, driving DsRed expression with the 2.2 $\mathrm{kb}$ bovine rhodopsin promoter (Matsuda and Cepko, 2004), was provided by Constance Cepko (Harvard Medical School, Boston, MA) via Addgene (Addgene plasmid 11156). cDNA for mouse R9AP (Martemyanov et al., 2003) and RGS9-1 (Makino et al., 1999) were used as templates for the generation of mutant constructs by PCR. A FLAG-tagged R7BPACT16 (Drenan et al., 2006) construct was kindly provided by Kendall Blumer (Washington University, St. Louis, MO) and an $\mathrm{N}$-terminally myc-tagged type II activin receptor (Gray et al., 2000) was provided by Wylie Vale (Salk Institute, La Jolla, CA).

Primers introducing $5^{\prime}$-AgeI sites and 3'-NotI sites were designed for each construct used in electroporation experiments (primer sequences are available upon request). To generate R9AP $\Delta$ CT31, a reverse primer adding a STOP codon after the codon for Gly-206 was used. To generate ggR9AP, a reverse primer was designed to introduce codons for the amino acids CCIIL after Gly-206 of R9AP. To tag RGS9-1 with the FLAG epitope, a reverse primer was used to append the FLAG nucleotide sequence immediately after the $3^{\prime}$ end of the RGS9-1 cDNA. To generate full-length FLAG-tagged R7BP, a reverse primer was designed to add the final 16 codons to the end of R7BP $\Delta$ CT16. Addition of the lipidated peptide from $\mathrm{G} \alpha$ o to RGS9-1 was accomplished by designing a forward primer to introduce codons for MGCTLSAEERA followed by a linker consisting of AKLTEER (McCabe and Berthiaume, 1999) just upstream of the coding sequence for RGS9-1. Addition of a transmembrane domain to RGS9-1 was achieved by deleting the codons for Glu-36 through Tyr-116 of the type II activin receptor (to remove most of its bulky extracellular domain) and then fusing the codon for Lys- 165 to the $5^{\prime}$ end of RGS9-1 using overhang extension PCR. The coding sequence for mCherry was similarly fused to the $3^{\prime}$ end of mouse rhodopsin cDNA. All DNA constructs were cloned between AgeI and NotI sites in pRho and fully sequence-verified.

Mouse strains and generation of transgenic lines. Procedures involving mice were performed in accordance with NIH guidelines and were approved by the Duke University and University of California, Davis, Institutional Animal Care and Use Committees. The $R 9 A P^{-{ }^{-}}$mouse strain with a targeted deletion of R9AP (Keresztes et al., 2004) and the transgenic mouse line overexpressing full-length R9AP (R9AP138) (Krispel et al., 2006) have been described previously. Experiments with wild-type mice used the C57BL/6 strain obtained from Charles River.

To generate transgenic mouse lines expressing mutant R9AP constructs, primers were designed to add $5^{\prime}$-SalI and $3^{\prime}$-BamHI restriction sites to the ends of the R9AP $\Delta$ CT31 and ggR9AP sequences. The PCR products were cloned between the SalI/BamHI restriction sites into a rod-specific expression vector carrying the $4.4 \mathrm{~kb}$ mouse rhodopsin promoter and a polyadenylation signal of the mouse protamine gene (Lem et al., 1991). Linear fragments were excised by digest with KpnI and NotI and were purified using the QIAGEN Gel Extraction kit. The DNA was injected into pronuclei of mouse embryos from superovulated FVB/ NHsd females by the Duke University Transgenic Mouse Facility. Potential transgenic founders were identified by the PCR analysis of tail DNA. Genotyping of R9AP $\Delta$ CT31 and ggR9AP was performed with the forward primer 5'-CCTGGAGTTGCGCTGTGGGAGC-3' recognizing the mouse rhodopsin promoter and the reverse primer $5^{\prime}$-AGCTAAGCTC CGGTCC-3' recognizing a sequence within R9AP; the PCR product is $\sim 300 \mathrm{bp}$ in length. To establish the final mouse lines, the founders were crossed with $R 9 A P^{-1-}$ mice. For experiments, we used mice of the $\mathrm{F}_{2}$ generation on the $R 9 A P^{-1-}$ background.

In vivo electroporation of mouse retinas. Retinal transfection of neonatal mice was performed as described by Matsuda and Cepko (2004). Briefly, following anesthetization of the mice on ice, the eyelid and sclera were punctured at the periphery of the eye using a 30 ga needle. A bluntend 32 ga needle on a Hamilton syringe was advanced through the defect until reaching the subretinal space, at which point $0.3-0.5 \mu$ l of concentrated plasmid DNA $(4 \mu \mathrm{g} / \mu \mathrm{l}$ of the construct of interest and $2 \mu \mathrm{g} / \mu \mathrm{l}$ DsRed or rhodopsin-mCherry to label transfected cells) was deposited. A tweezer-type electrode (BTX) was placed over the mouse's head with the positive electrode overlying the injected eye. Five $80 \mathrm{~V}$ pulses of $50 \mathrm{~ms}$ duration were applied using an ECM830 square pulse generator (BTX). The neonates were returned to their dam and allowed to develop until harvesting of retinal tissue at postnatal day 21.

Western blot analysis. Mouse retinas were excised and placed in lysis buffer consisting of PBS with $0.5 \%$ NP-40 and a protease inhibitor mixture (Complete Mini EDTA-free tablets; Roche). Following homogenization with a motorized pestle and two $5 \mathrm{~s}$ sonicator pulses, the lysates were clarified at $5000 \times g$, and protein concentrations were measured using a Bio-Rad DC protein assay. Samples were diluted to equal protein concentrations in SDS-PAGE sample buffer and boiled. Retinal proteins were separated on 4-20\% SDS-PAGE gels, transferred onto PVDF membranes, blocked in Odyssey blocking buffer (LI-COR), and blotted with primary antibodies. After incubating with appropriate secondary antibodies conjugated to Alexa Fluor 680 (Invitrogen) or IR800 (Invitrogen), blots were scanned on an Odyssey imaging system (LI-COR). Quantitative Western blotting to compare the R9AP and RGS9-1 contents of wild-type and transgenic retinal samples after normalizing to rhodopsin concentration was performed as previously described (Krispel et al., 2006). Rhodopsin concentration was determined by difference spectroscopy at $500 \mathrm{~nm}$ before and after bleaching the sample, as described by Bownds et al. (1971) using the molar extinction coefficient of 40,500.

To analyze soluble and membrane fractions from mouse retinas, dissected retinal tissue was placed in deionized water with protease inhibitors. After mechanical homogenization and brief sonication, each sample was centrifuged at $240,000 \times g$ for $30 \mathrm{~min}$ on a TLA 120 rotor on a tabletop Optima Max-E ultracentrifuge (Beckman Coulter). Afterward, the supernatant was removed and mixed with sample buffer, representing the soluble fraction. The pellet was resuspended in deionized water with $1 \%$ Triton X-100 to solubilize membranes, followed by another 
centrifugation step as above to pellet insoluble material. The supernatant was removed and mixed with sample buffer, representing the membrane fraction. After boiling, proteins were separated on 4-20\% SDS-PAGE gels, transferred onto PVDF membranes, blotted, and imaged.

Immunofluorescence. Posterior eyecups from mouse eyes were obtained by microdissection, fixed in 4\% paraformaldehyde in PBS for 15 min at room temperature, cryoprotected in $30 \%$ sucrose for $1 \mathrm{~h}$ at $4^{\circ} \mathrm{C}$, and embedded in OCT over liquid nitrogen. Twelve micrometer frozen sections were collected, blocked in $5 \%$ goat serum in PBS with $0.1 \%$ Triton X-100 for $1 \mathrm{~h}$, incubated in primary antibody diluted in blocker for $1.5 \mathrm{~h}$, washed three times in PBS with $0.1 \%$ Triton, and stained for $1 \mathrm{~h}$ with goat secondary antibodies conjugated to Alexa Fluor 488 or 594 (Invitrogen) and $10 \mu \mathrm{g} / \mathrm{ml}$ Hoechst 33342 (Invitrogen) to label nuclei. Retinal flat mounts were prepared by gently detaching the retinas from posterior eyecups and fixing them in paraformaldehyde as above. The free-floating retinas were blocked and stained in Eppendorf tubes using the solutions described above. Several radial incisions were made at the periphery of the retinas, allowing them to be placed flattened onto glass slides with the photoreceptor layer facing up. All samples were mounted with Fluoromount (EMS) under glass coverslips and visualized using a Nikon Eclipse 90i microscope and a C1 confocal scanner controlled by EZ-C1, version 3.10, software (Nikon).

Single-cell recordings. Suction electrode recordings from the outer segments of intact mouse rods were performed as previously described (Gross and Burns, 2010). Dark-adapted retinas were stored in L-15 supplemented with BSA $(1 \mathrm{mg} / \mathrm{ml})$ and $10 \mathrm{~mm}$ glucose, and stored on ice. Recordings were performed in oxygenated, bicarbonate-buffered Locke's solution supplemented with $10 \mathrm{~mm}$ glucose at $35-37^{\circ} \mathrm{C}$. The suction pipette contained HEPES-buffered Locke's solution, $\mathrm{pH}$ 7.4, connected to a calomel electrode via an agar bridge. An agar bridge also connected the bath to the opposite calomel half cell.

Brief $(10 \mathrm{~ms}, 500 \mathrm{~nm})$ flashes of calibrated strength were used to elicit electrical responses, which were amplified (Axopatch 200B; Molecular Devices), filtered at $30 \mathrm{~Hz}$ with an eight-pole Bessel (Frequency Devices), and digitized at $200 \mathrm{~Hz}$ using custom-written acquisition procedures in IgorPro (Wavemetrics). Responses to a large number $(>30)$ of dim flashes were averaged and used to determine the mean time to peak and integration time if the peak amplitude was $<20 \%$ of the maximal response amplitude (dark current). Variance to mean analysis of the dim flash responses was used to calculate the average single photon response for each cell, and saturating flash responses were used to calculate the dominant time constant of recovery $\left(\tau_{\mathrm{D}}\right)$ as previously described (Krispel et al., 2006; Gross and Burns, 2010).

\section{Results}

Physical interaction between R9AP and RGS9-1 is not sufficient for protection of the transducin GTPase-activating complex in rods

R9AP exhibits high structural similarity to the SNARE proteins involved in vesicular fusion (Keresztes et al., 2003; Martemyanov et al., 2003). It consists of an N-terminal trihelical bundle, followed by a fourth helical region known as a SNARE homology domain, and finally a C-terminal transmembrane domain allowing the rest of the protein to face the cytosol (Fig. $1 A$ ). It has been shown that the interaction between R9AP and RGS9-1 - G $\beta 5$ is mediated by the trihelical bundle of R9AP; neither the transmembrane domain (Hu and Wensel, 2002) nor the SNARE homology domain (Baker et al., 2006) is required for this interaction. The trihelical bundle of R9AP binds to an interface formed by the N-terminal DEP domain of RGS9-1 and its adjacent R7 homology domain, with contributions from G $\beta 5$ (Martemyanov et al., 2003; Porter et al., 2010; Masuho et al., 2011).

To test the hypothesis that it is solely the physical interaction between R9AP and RGS9-1 - G $\beta 5$ that is required to protect RGS9-1 - G $\beta 5$ from proteolysis, we generated a transgenic mouse line carrying a mutant R9AP construct with a deletion of the last 31 aa, thereby eliminating its transmembrane domain

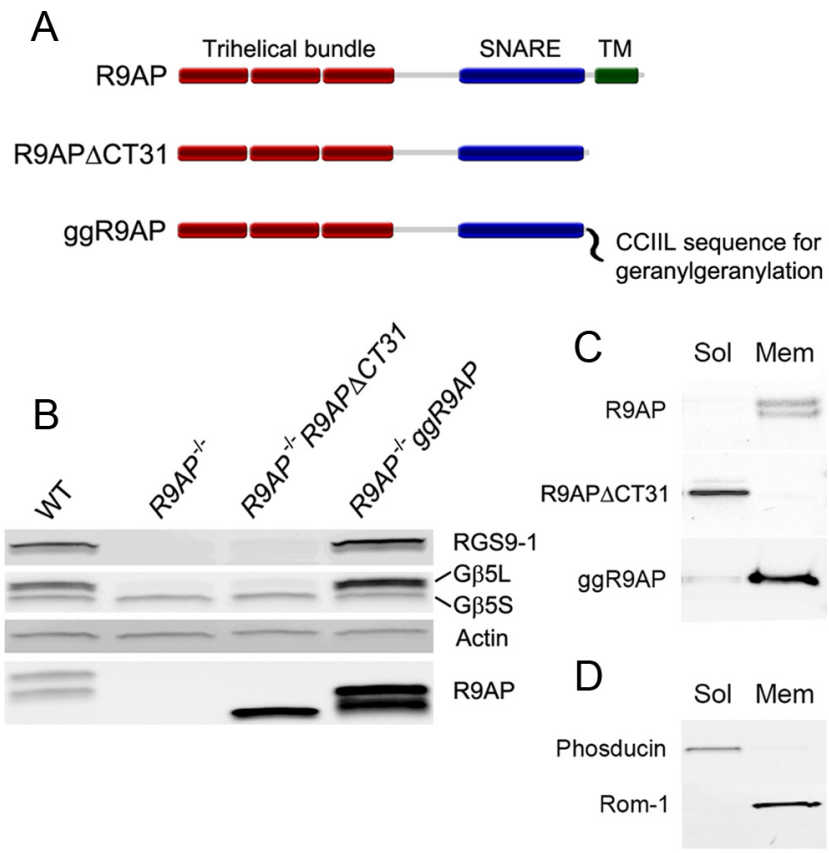

Figure 1. R9AP must be membrane bound to stabilize RGS9-1 $\cdot G \beta 5$. $A$, Diagram of the R9AP protein (top) and the mutant proteins R9AP $\Delta$ CT31 (middle) and ggR9AP (bottom), highlighting the major protein domains. R9AP consists of four helical domains, the first three of which form a trihelical bundle that binds RGS9-1, and the fourth of which is a SNARE homology domain. The transmembrane domain is found at the $C$-terminal tail of the protein and has been deleted in R9AP $\triangle$ CT31 and replaced in ggR9AP by a CAAX box enabling geranylgeranylation. $\boldsymbol{B}$ Western blot analysis of mouse retinal extracts. Ten micrograms of total protein for wild-type (WT), $R 9 A P^{-1-}, R 9 A P^{-1-} R 9 A P \Delta C\left(T 31\right.$, and $R 9 A P^{-/-}$ggR9AP mouse retinal lysates were loaded in each lane, and the membrane was probed for each of the proteins indicated on the right with specific antibodies. C, Membrane and soluble retinal fractions from WT, R9AP ${ }^{-1-}$ $R 9 A P \triangle C T 31$, and $R 9 A P^{-1-}$ ggR9AP mouse retina homogenates immunostained for R9AP. D Wild-type retinal fractions from the mouse in $C$ additionally stained for the soluble protein phosducin and the outer segment disc membrane protein Rom-1.

("R9AP $\Delta$ CT31"; Fig. $1 A$ ). This construct was expected to be fully soluble, because R9AP has no predicted lipid modifications. As a control, we generated a second transgenic line carrying a modified version of R9AP $\triangle$ CT31 that was designed to be membrane-anchored by the alternative means of geranylgeranylation ("ggR9AP"; Fig. $1 A$ ) via addition of a C-terminal CAAX box (CCIIL). The transgenes were placed under the transcriptional control of the $4.4 \mathrm{~kb}$ mouse rhodopsin promoter to drive expression in rod photoreceptors (Lem et al., 1991).

Potential founders were identified and crossbred with $R 9 \mathrm{AP}^{-1-}$ mice, yielding two lines expressing R9AP $\Delta \mathrm{CT} 31$ and five lines expressing ggR9AP. Western blot analysis of lysates from transgenic mouse retinas demonstrated that both mutant proteins in all lines were expressed at higher levels than R9AP in wild-type retinas, as demonstrated for representative retinas in Figure $1 B$. Due to the loss of the transmembrane domain, both R9AP $\triangle C T 31$ and ggR9AP migrated slightly faster than fulllength R9AP. The R9AP $\triangle$ CT31 protein ran as a single band, unlike wild-type R9AP, which exists as a doublet due to variable phosphorylation ( $\mathrm{Hu}$ and Wensel, 2002; Martemyanov et al., 2003). This observation may indicate that the kinase(s) responsible for phosphorylating R9AP are confined to the cellular membranes of the rods and cannot access R9AP in the cytosolic space. Despite robust expression of R9AP $\Delta$ CT31, there was no evidence of rescue of RGS9-1 or of the long isoform of G $\beta 5$ in $R 9 A P^{-/-}$ $R 9 A P \Delta C T 31$ retinas [the short isoform of $\mathrm{G} \beta 5$ is expressed in other retinal cell types (Watson et al., 1996), and its expression 


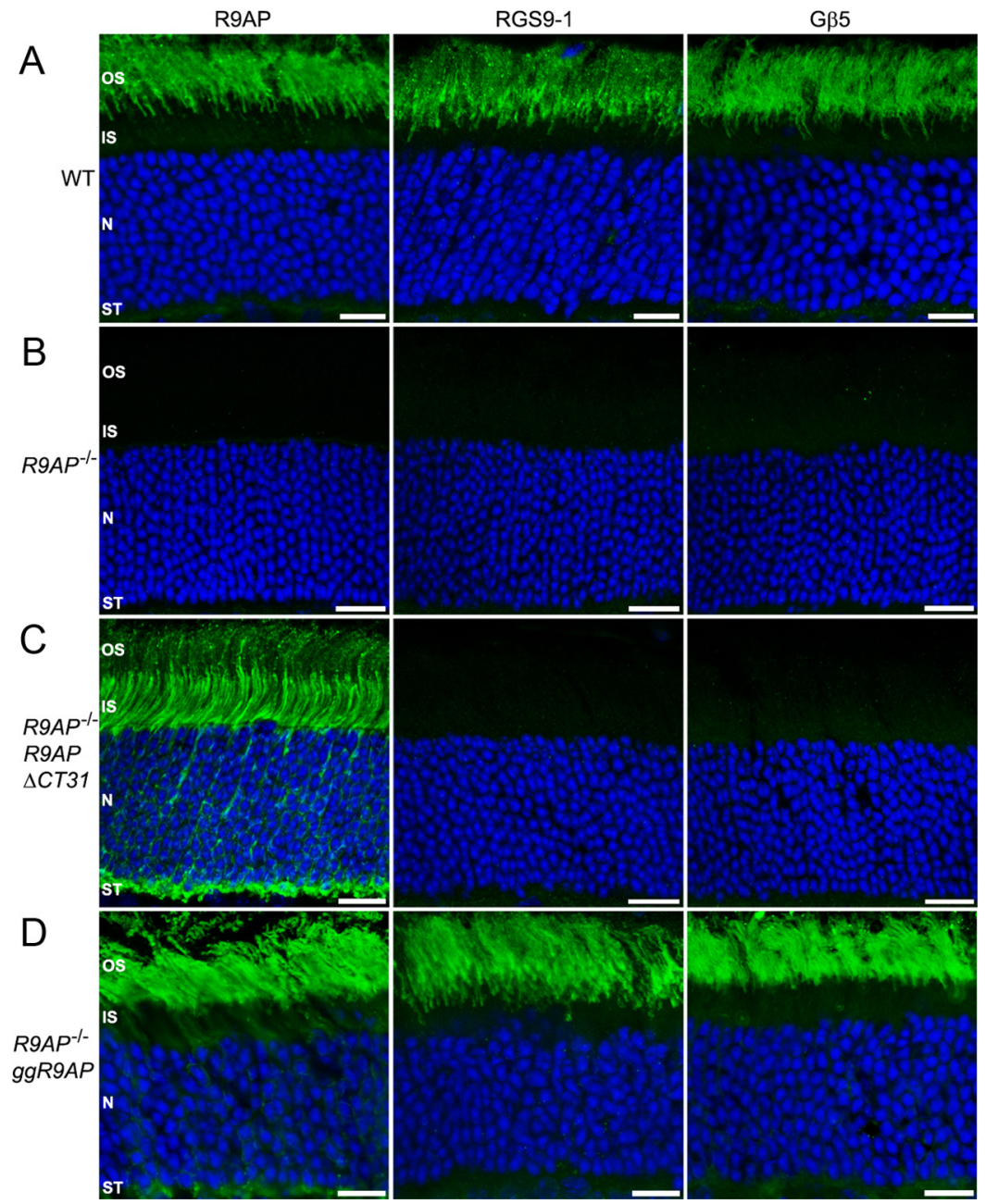

Figure 2. Immunohistochemical analysis of transducin GTPase-activating complex expression and localization. Retinal cross sections from the mouse lines indicated on the left were immunostained for R9AP, RGS9-1, and G $\beta 5$ (green), as indicated above each panel. Nuclei are counterstained with Hoechst 33342 (blue). Subcellular compartments of photoreceptor cells are labeled as follows: OS, outer segment; IS, inner segment; N, nuclear region; ST, synaptic terminal. Scale bar, $10 \mu \mathrm{m}$.

level is not influenced by R9AP (Krispel et al., 2006)]. In contrast, $R 9 A P^{-1-}$ ggR9AP retinas demonstrated complete rescue of RGS9-1 - G $\beta 5$. To confirm that the capacity of the R9AP mutants to protect RGS9-1 $\cdot$ G $\beta 5$ truly reflected their ability to attach to cellular membranes, we performed fractionation of mouse retina samples (Fig. 1C,D). As expected, the R9AP $\Delta$ CT31 protein was found exclusively within the soluble fraction, while ggR9AP, like wild-type R9AP, partitioned almost entirely to the membrane fraction.

Immunolabeling of retinal cross sections from these mice supported the above findings. While wild-type retinas demonstrated prominent outer segment staining for all three components of the GTPase-activating complex, none of them was detectable in the photoreceptors of $R 9 A P^{-/-}$mice (Fig. $2 A, B$ ). In $R 9 A P^{-/}$ ${ }^{-}$R9AP $\triangle C T 31$ mice, the mutant R9AP $\Delta$ CT31 construct exhibited a localization pattern characteristic of a soluble protein, filling the cytosolic space of the rod inner segment, nuclear region, and synaptic terminal, while failing to localize strongly to the membrane-rich outer segment (Fig. 2C). Consistent with the Western blot analysis, there were no discernible differences between the $R 9 A P^{-1-} R 9 A P \Delta C T 31$ retinas and the $R 9 A P^{-1-}$ retinas when retinal sections were immunostained for RGS9-1 or $\mathrm{G} \beta 5$. In $R 9 A P^{-/-} g g R 9 A P$ retinas, the mutant construct was expressed very strongly, with the vast majority of the ggR9AP pro- tein localizing to the rod outer segment (Fig. 2D). Both RGS9-1 and G $\beta 5$ were protected in these rods and found almost exclusively in the outer segment. Collectively, these data indicate that the ability to bind RGS9-1 - G $\beta 5$ is not sufficient for R9AP to perform its protective function; R9AP must also have a means of membrane attachment.

\section{The ability of RGS anchor protein R7BP to protect \\ RGS9-1 - G $\beta 5$ outside of rod outer segments does not reflect superior masking of labile RGS9-1 residues}

While membrane recruitment of RGS9$1 \cdot \mathrm{G} \beta 5$ appeared to be a key component of the protective function of R9AP, it remained unclear whether sequestering the complex in outer segment membranes was specifically required. In approaching this question, the example of the RGS anchor protein R7BP seemed particularly instructive. R7BP shares considerable structural homology with R9AP and has similarly been shown to bind RGS9-1 • G $\beta 5$ (Drenan et al., 2005; Martemyanov et al., 2005). When heterologously expressed in rods, R7BP was found to be excluded from outer segments and instead localized to the plasma membrane in the rest of the cell (Cao et al., 2010). There, it successfully bound and stabilized RGS9-1 $\cdot$ G $\beta 5$, suggesting that recruitment of RGS9-1 - G $\beta 5$ to any membrane in rods is sufficient for an anchor protein to provide protection. However, there is a caveat: despite the structural similarities between R7BP and R9AP, they differ in their mechanism of binding to RGS9$1 \cdot$ G $\beta 5$. Unlike R9AP, R7BP uses not just its trihelical bundle but also its SNARE homology domain for binding (Anderson et al., 2007b). This opens the possibility that R7BP might mask the labile regions of RGS9-1 more effectively than R9AP, an idea consistent with the finding that a soluble R7BP mutant protected the RGS9 splice variant, RGS9-2, from proteolysis in cell culture (Anderson et al., 2007a). We therefore sought to further investigate the mechanism by which the protection of RGS9-1 - G $\beta 5$ outside of rod outer segments is accomplished by heterologously expressed R7BP.

At this point, we decided to switch from conventional transgenics to in vivo electroporation of mouse retinas (Matsuda and Cepko, 2004). This gene delivery method has the distinct advantage of allowing analysis of a large number of DNA constructs in an extremely time-efficient manner. Neonatal mouse retinas were transfected with plasmid DNA containing the $2.2 \mathrm{~kb}$ bovine opsin promoter to express constructs specifically in rods, allowing immunohistochemical analysis to be performed as soon as the rod cells reached maturity around postnatal day 21 . We validated this strategy by replicating the results of our previous experiments performed with conventional transgenic animals. $R 9 A P^{-1-}$ rods were transfected with wild-type R9AP, R9AP $\triangle$ CT31, or ggR9AP, and the distribution of R9AP and 


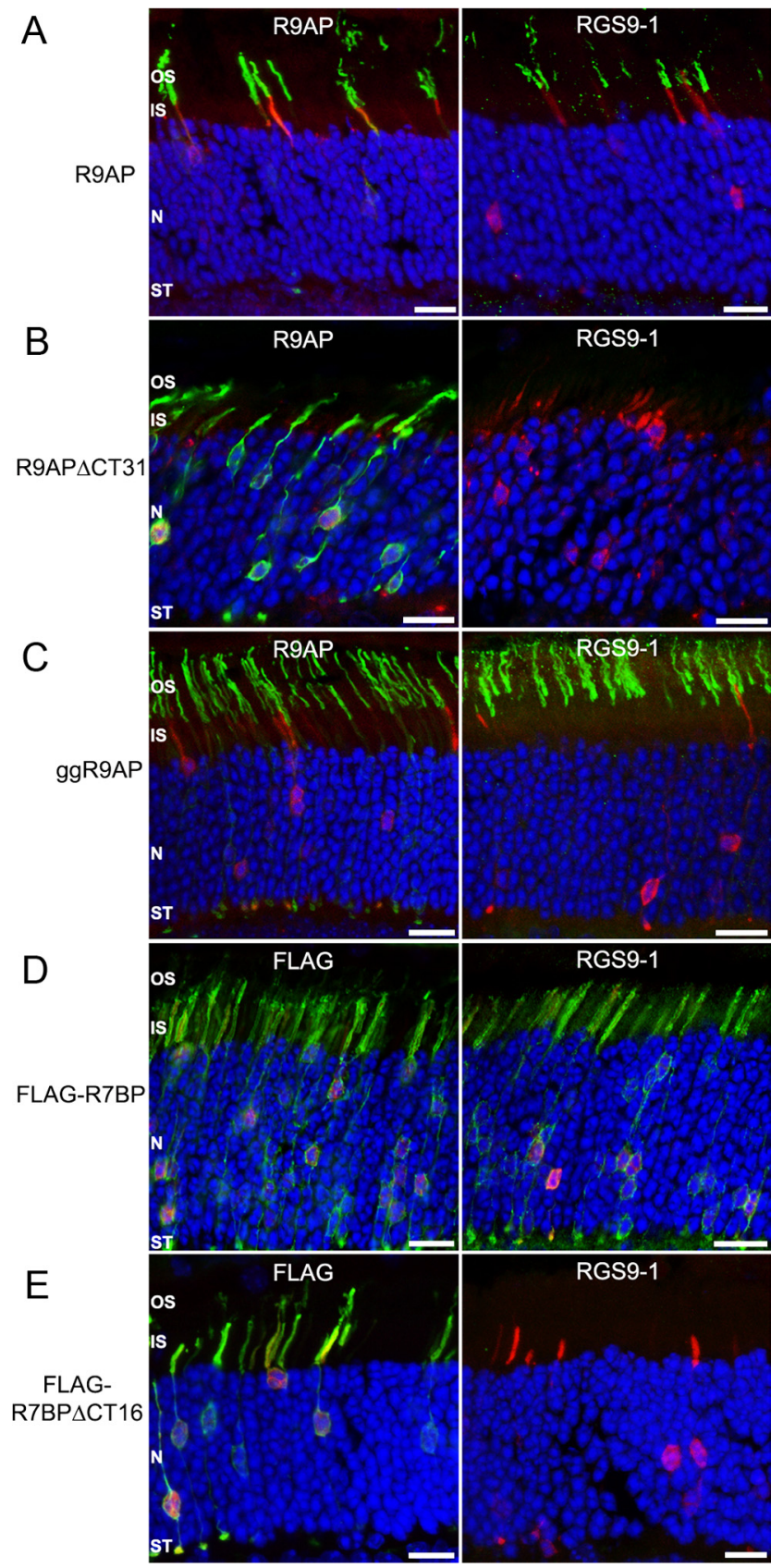

Figure 3. Anchor protein R7BP requires membrane attachment for protection of RGS9-1 in rods. $R 9 A P^{-1-}$ mouse retinas were transfected with wild-type R9AP $(\boldsymbol{A}), \operatorname{R9AP} \Delta \mathrm{CT} 31(\boldsymbol{B})$, ggR9AP (C), FLAG-tagged wild-type R7BP (D), or FLAG-tagged R7BP $\Delta$ CT16 (E). Cross sections were stained for R9AP or FLAG (green; left panels) or for endogenous RGS9-1 (green; right panels). In all cases, retinas were cotransfected with DsRed so that successfully transfected regions of retina could be identified (red). Photoreceptor compartments are labeled as in Figure 2. Scale bar, $10 \mu \mathrm{m}$.

RGS9-1 was subsequently analyzed by immunolabeling each sample (Fig. $3 A-C$ ). Individual cells that had been successfully transfected exhibited robust expression of the R9AP constructs, which localized predominantly to rod outer segments in the cases of R9AP and ggR9AP, but yielded a pattern consistent with a cytoplasmic distribution in the case of R9AP $\Delta$ CT31. Rescue of RGS9-1 was readily observed in cells transfected with R9AP and ggR9AP, but not R9AP $\Delta$ CT31. Each of these results was a precise match of the corresponding data in Figure 2 achieved with transgenic mice.
We proceeded to transfect retinas from $R 9 A P^{-1-}$ mice with FLAG-tagged versions of either wild-type R7BP or a truncated R7BP mutant lacking the last $16 \mathrm{C}$-terminal residues (R7BP $\Delta \mathrm{CT} 16)$. This mutation preserved all domains required for RGS9-1 - G $\beta 5$ binding (Anderson et al., 2007b) while eliminating both the palmitoylation site mediating membrane attachment and a polybasic region directing palmitoylation-deficient R7BP to nuclei (Drenan et al., 2006). This ensured that the mutant construct would localize to the cytosol of the rod. As shown in Figure $3 D$, wild-type R7BP localized to the rod plasma membrane, except for that of the outer segment; this localization pattern is in complete accordance with that reported for the transgenic mouse model heterologously expressing R7BP in rods (Cao et al., 2010). Also in agreement, R7BP expression in transfected cells successfully rescued RGS9-1. In contrast, the soluble R7BP mutant, R7BP $\Delta$ CT16, failed to rescue RGS9-1 expression (Fig. 3E), just as we had observed with soluble R9AP. This indicates that, despite its different binding mechanism, R7BP also requires membrane attachment to protect RGS9-1 in rods. In light of this finding, it would seem most plausible that simple membrane recruitment of RGS9-1 - G $\beta 5$ serves as the basic mechanism by which R9AP stabilizes the complex and that delivering RGS9-1 - G $\beta 5$ to rod outer segments is not inherent to the protective function of R9AP.

Direct attachment of RGS9-1 to membranes obviates the need for protection by membrane anchor proteins

While the previous experiments demonstrated that membrane recruitment is necessary for protection of the GTPase-activating complex in rods, it remained unclear whether this mechanism is sufficient or whether a physical interaction between RGS9-1 and $\mathrm{R} 9 \mathrm{AP}$ (or R7BP) is also required to mask destabilizing residues. Therefore, we assessed whether directly attaching RGS9-1 to membranes would be sufficient for its stable expression in rods.

We decided to test two alternative means of constructing a membrane-bound RGS9-1 protein: addition of a randomly chosen transmembrane domain ("tm-RGS9-1") and acylation ("acyl-RGS9-1"). To generate tm-RGS9-1, we exploited the transmembrane domain of the activin receptor, a single-pass membrane protein containing a well defined signal sequence and oriented with respect to the membrane such that its $\mathrm{N}$ terminus is extracellular and its $\mathrm{C}$ terminus is intracellular. This orientation allowed us to append the signal sequence and transmembrane domain of the activin receptor directly to the $\mathrm{N}$ terminus of RGS9-1, ensuring that RGS9-1 would remain on the cytosolic face of the membrane. To generate the acyl-RGS9-1, we added the first 11 aa from the $\alpha$-subunit of the G-protein $\mathrm{G}_{\mathrm{o}}$ to the $\mathrm{N}$ terminus of RGS9-1. This peptide contains sites for both myristoylation and palmitoylation and has been shown to be sufficient to attach GFP to membranes in cultured cells (McCabe and Berthiaume, 1999). $R 9 A P^{-1-}$ retinas were transfected with wild-type RGS9-1, tm-RGS9-1, and acyl-RGS9-1, all three of which were C-terminally FLAG-tagged (Fig. $4 A-C$ ). While the wild-type RGS9-1 construct predictably failed to stably express in $R 9 A^{-/-}$rods, both membrane-associated constructs were easily detected by immunohistochemistry in transfected rods. Interestingly, both tm-RGS9-1 and acyl-RGS9-1 localized to the plasma membrane of the rod inner segment, nuclear region, and synaptic terminal but were absent from the membrane-rich outer segment (Fig. $4 B, C$ ). Their total exclusion from the outer segment is most easily appreciated in rods cotransfected with a rhodopsin-mCherry fusion construct to specifically label this compartment (shown for acyl-RGS9-1 in Fig. 4D). Together, 


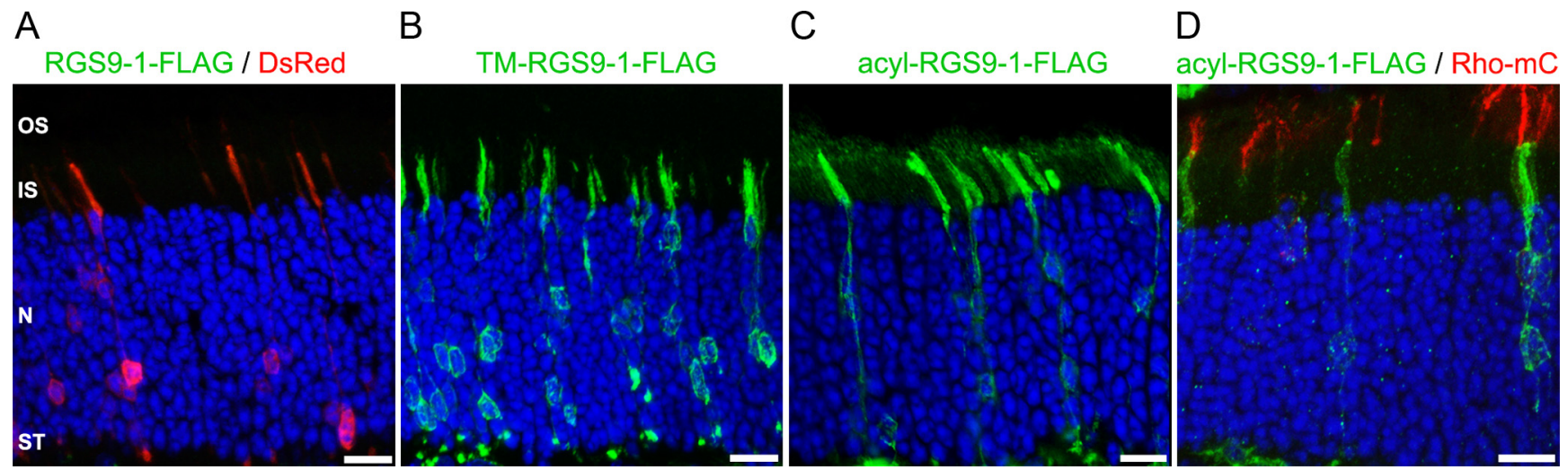

Figure 4. Direct membrane attachment of RGS9-1 is sufficient for its stable expression but not rod outer segment localization. $R 9 A P^{-1-}$ retinas were transfected with the following FLAG-tagged constructs: RGS9-1 (A), RGS9-1 endowed with an N-terminal transmembrane domain ( $\boldsymbol{B}$ ), and acylated RGS9-1 (C, $\boldsymbol{D})$. Retinal cross sections were stained for FLAG (green). In $\boldsymbol{A}$, cotransfected DsRed (red) is shown to mark successfully transfected rods. In $\boldsymbol{D}$, cotransfected rhodopsin-mCherry (red) labels rod outer segments. Photoreceptor compartments are labeled as in Figure 2. Scale bar, $10 \mu \mathrm{m}$.
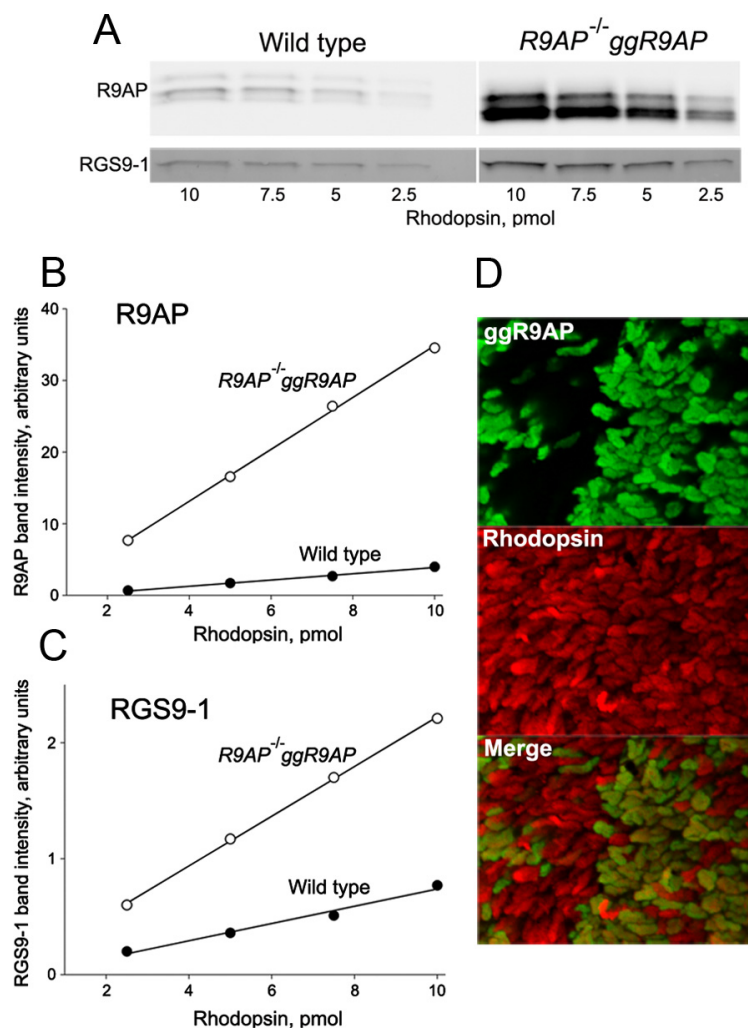

Figure 5. Assessment of ggR9AP expression in transgenic mouse retinas. $A$, Representative Western blot analysis comparing the expression of R9AP and RGS9-1 in the retinas of wild-type and $R 9 A P^{-1-}$ ggR9AP (line 1) mice. For each mouse line, retinal lysates containing 10, 7.5, 5, and $2.5 \mathrm{pmol}$ of rhodopsin (measured as described in Materials and Methods) were loaded on the same gel and probed with the corresponding primary antibodies. $B, C$, Signal intensities of each band from the blot in $A$ were measured and plotted as a function of rhodopsin content. The relative expression of each protein between the two mice was calculated as the ratio of the slopes of the linear regressions. The $R 9 A P^{-/}-g g R 9 A P$ retina overexpressed R9AP by 8.3 -fold (B) and RGS9-1 by 2.9-fold (C. D, Immunolabeling of a $R 9 A P^{-1}{ }^{-}$ggR9AP retinal flat mount for R9AP (green) and rhodopsin (red) reveals patches of photoreceptor outer segments lacking R9AP expression. Scale bar, $10 \mu \mathrm{m}$.

these data indicate that membrane recruitment is the only mechanism relevant to the protection of RGS9-1 from proteolysis. However, the role of R9AP in targeting the GTPase-activating complex to outer segments cannot be replaced by direct membrane attachment of RGS9-1.
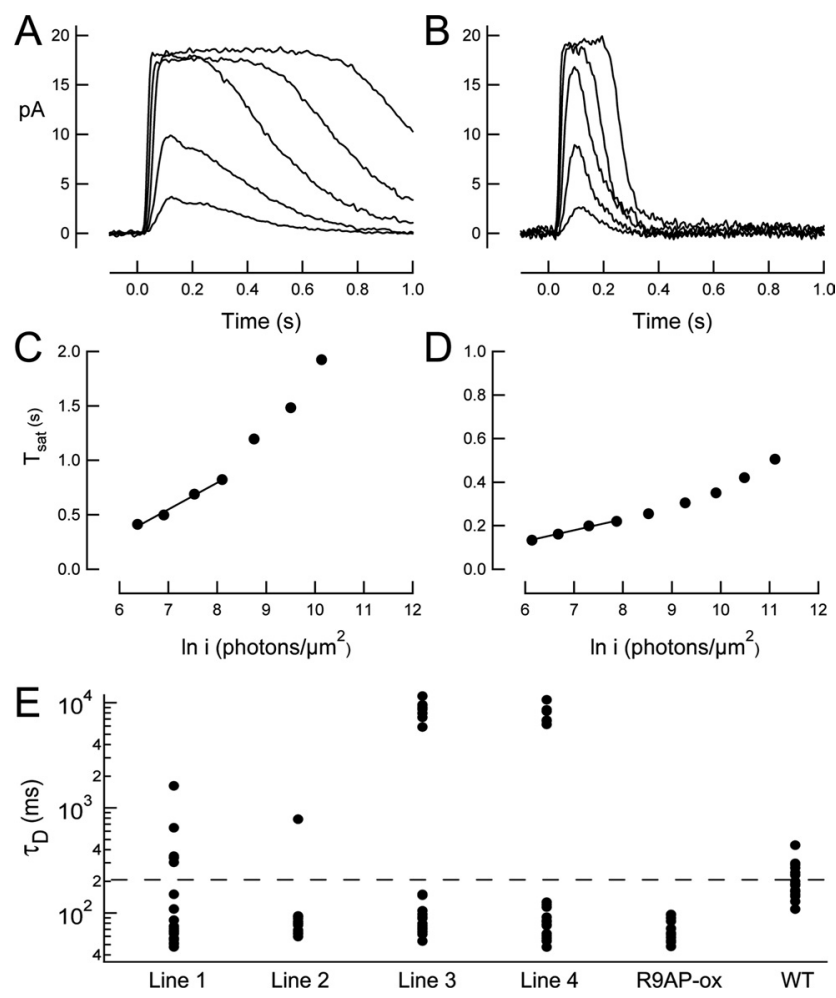

Figure 6. Overexpression of ggR9AP causes faster than normal photoresponse recovery. $\boldsymbol{A}$, $\boldsymbol{B}$, Representative families of average responses to flashes that ranged from 11 to 1484 photons $\mu \mathrm{m}^{-2}$ in roughly fourfold increments for a wild-type rod $(\boldsymbol{A})$ and a ggR9AP-overexpressing rod from line $1(\boldsymbol{B}) . \boldsymbol{C}, \boldsymbol{D}$, Plots of the time that a bright flash response remained in saturation $\left(T_{\mathrm{sat}}\right)$ as a function of the natural $\log$ of the flash strength $(\operatorname{In} i)$ for the cells shown in $\boldsymbol{A}$ and $\boldsymbol{B}$. The slope of the initial, linear portion of this relation represents $\tau_{D}$, the dominant time constant of recovery, which reflects the rate of GTP hydrolysis on transducin (Krispel et al., 2006). The $\tau_{D}$ values were 245 and $51 \mathrm{~ms}$ for wild-type and $R 9 A P^{-/-}$ggR9AP rods, respectively. E, Variability of $\tau_{D}$ across transgenic lines expressing ggR9AP. All $R 9 A P^{-\prime}{ }^{-}$ggR9AP lines showed cell-to-cell variability in their ggR9AP expression and $\tau_{D}$ values. The data for a conventional R9APoverexpressing line, R9AP138 (labeled as R9AP-ox), and wild-type mice are also shown. The dotted horizontal line represents the mean $\tau_{D}$ value for wild-type rods (207 ms; Table 1).

\section{Overexpression of ggR9AP speeds photoresponse recovery in transgenic rods}

One of the transgenic animals generated in this study, the ggR9AP mouse, provided us with an opportunity to study the functional properties of the GTPase-activating complex when 
Table 1. Characteristics of dark-adapted transgenic mouse rods with $\tau_{\mathrm{D}}$ less than the mean wild-type mouse value

\begin{tabular}{|c|c|c|c|c|c|c|c|}
\hline & Dark current (pA) & Time to peak (ms) & $\tau_{\text {rec }}(\mathrm{ms})$ & Integration time (ms) & Elementary amplitude (pA) & $I_{0}\left(\text { photons } / \mu \mathrm{m}^{2}\right)^{a}$ & $\tau_{\mathrm{D}}(\mathrm{ms})$ \\
\hline Wild type & $11.9 \pm 0.6(23)$ & $105 \pm 5(22)$ & $168 \pm 13(20)$ & $249 \pm 22(22)$ & $0.62 \pm 0.08(17)$ & $52.0 \pm 2.7(22)$ & $207 \pm 16(21)$ \\
\hline Wild type, $\tau_{\mathrm{D}}<207 \mathrm{~ms}$ & $11.9 \pm 0.7(16)$ & $100.3 \pm 4.5(15)$ & $143.9 \pm 10.8(13)$ & $211.7 \pm 16.1(15)$ & $0.66 \pm 0.09(14)$ & $54.0 \pm 3.3(15)$ & $167 \pm 7.7(14)$ \\
\hline ggR9AP, line 1 & $15.2 \pm 1.0(18)$ & $93.3 \pm 3.5(18)$ & $74.2 \pm 9.4(16)$ & $130.9 \pm 9.5(18)$ & $0.60 \pm 0.09(16)$ & $78.6 \pm 5.2(18)$ & $69.6 \pm 7.0(16)$ \\
\hline ggR9AP, line 2 & $12.8 \pm 0.9(17)$ & $97 \pm 6(17)$ & $98 \pm 11(11)$ & $132.2 \pm 10.6(18)$ & $0.69 \pm 0.16(13)$ & $75.2 \pm 6.1(17)$ & $78.0 \pm 3.0(17)$ \\
\hline ggR9AP, line 3 & $15.1 \pm 1.0(19)$ & $114.2 \pm 5.13(19)$ & $122 \pm 14(18)$ & $179.9 \pm 14.1(19)$ & $0.93 \pm 0.11(14)$ & $56.8 \pm 3.7(19)$ & $79.2 \pm 5.4(18)$ \\
\hline ggR9AP, line 4 & $12.2 \pm 0.8(15)$ & $81.9 \pm 5.3(15)$ & $71.4 \pm 8.9(14)$ & $146.6 \pm 29.6(15)$ & $0.37 \pm 0.05(13)$ & $104.4 \pm 10.3(15)$ & $77.8 \pm 6.9(15)$ \\
\hline R9AP-ox, line 138 & $12.8 \pm 0.9(12)$ & $91 \pm 5(12)$ & $58 \pm 8(12)$ & $128 \pm 13(12)$ & $0.62 \pm 0.14(10)$ & $64.6 \pm 4.3(12)$ & $70.2 \pm 5.2(12)$ \\
\hline
\end{tabular}

${ }^{a}$ Flash strength that elicited a half-maximal response; the number of analyzed cells is indicated in parentheses.

attached by an alternative means to outer segment disc membranes. As shown above, both ggR9AP and RGS9-1 - G $\beta 5$ were faithfully delivered to the outer segments of $R 9 A P^{-/-} g g R 9 A P$ mice. Of the five transgenic lines we obtained, one (line 5) expressed ggR9AP at such high levels that retinal degeneration was induced (data not shown). Quantitative Western blotting showed that the other four lines expressed ggR9AP at levels ranging from 2.7- to 8.3-fold higher than R9AP protein expression in wild-type mice (for a representative analysis, see Fig. 5A,B). RGS9-1 was also overexpressed in these mice (Fig. $5 A, C$ ), with levels ranging between 1.7- and 4.9-fold above the wild-type RGS9-1 content. We noted, however, that measurements of protein content had unusual variability among mice of the same line, leading us to suggest that rods from ggR9AP mice exhibit mosaicism in their expression of the transgene, as occasionally observed in transgenic lines using this promoter (Lem et al., 1991) and encountered in one of our recent studies (Martemyanov et al., 2008). Staining of $R 9 A P^{-/-}$ggR9AP retinal flat mounts for $\mathrm{R} 9 \mathrm{AP}$ revealed that this was indeed the case, as patches of rhodopsin-positive outer segments without R9AP expression were easily identified (Fig. 5D).

To assess the ability of ggR9AP $\cdot \mathrm{RGS9}-1 \cdot \mathrm{G} \beta 5$ to inactivate transducin in intact rods, we used suction electrodes to record their responses to brief flashes of varying intensity. We were particularly interested in assessing the rate of response recovery from excitation, a parameter directly regulated by the GTPaseactivating complex. An important property of the photoresponse is that the rate of recovery observed over a broad range of light stimulus intensities is determined by a single, dominant time constant, $\tau_{\mathrm{D}}$ (Pepperberg et al., 1992; Pugh, 2006). $\tau_{\mathrm{D}}$ reflects the slowest (rate-limiting) biochemical step in phototransduction cascade inactivation, identified in wild-type rods as transducin inactivation via GTP hydrolysis (Krispel et al., 2006). Therefore, the value of $\tau_{\mathrm{D}}$ in our mice would provide the best measure of ggR9AP $\cdot$ RGS9- $1 \cdot$ G $\beta 5$ activity.

Recordings from rods of each of the four different transgenic lines were consistent with mosaic ggR9AP - RGS9-1 - G $\beta 5$ expression (Fig. 6). For each line, the light responses from individual rods varied dramatically in the speed with which the current recovered to baseline, with $\tau_{\mathrm{D}}$ ranging from $\sim 40 \mathrm{~ms}$ to $\sim 10 \mathrm{~s}$. Unlike wild-type rods that have a recovery time constant of $\sim 200$ ms (Fig. $6 A, C$ ), most $R 9 A P^{-/-} g g R 9 A P$ rods had time constants that were considerably shorter (Fig. $6 B, D$ ), consistent with a higher expression level of the transducin GTPase-activating complex (Krispel et al., 2006). In roughly one-third of the rods from two mouse lines (Fig. 6 E), the flash responses were indistinguishable from those of rods completely lacking the GTPase-activating complex $\left[\tau_{\mathrm{D}} \sim 10 \mathrm{~s}\right.$ (Chen et al., 2000; Krispel et al., 2003; Keresztes et al., 2004)]. The other two lines had individual rods with intermediate $\tau_{\mathrm{D}}$ values.

It was interesting to compare the $\tau_{\mathrm{D}}$ values in rods expressing high levels of ggR9AP $\cdot$ RGS9-1 $\cdot$ G $\beta 5$ with those from transgenic line R9AP138 (Fig. 6E) homogeneously overexpressing the normal R9AP - RGS9-1 • G $\beta 5$ complex at a level approximately fourfold higher than in wild-type mice (Krispel et al., 2006). However, because of the cell-to-cell variability in the expression level of ggR9AP $\cdot \operatorname{RGS9-1} \cdot \mathrm{G} \beta 5$, it made little sense to average $\tau_{\mathrm{D}}$ values across all cells from each line. Instead, we averaged $\tau_{\mathrm{D}}$ values only among the cells that clustered below the average $\tau_{\mathrm{D}}$ value of wildtype rods ( $207 \mathrm{~ms}$; Fig. 6 E, dotted line). This subset of fastest cells in all lines showed average $\tau_{\mathrm{D}}$ values that approached but did not fall below those of rods overexpressing the normal R9AP - RGS9$1 \cdot \mathrm{G} \beta 5$ complex (Table 1 ). Likewise, other kinetic features of the responses that also depend upon the GTPase activity, such as the time to peak, dim flash time constant of recovery $\left(\tau_{\text {rec }}\right)$, and integration time, were decreased in the $R 9 A P^{-/} g g R 9 A P$ rods. Other photoresponse characteristics were not systematically different in the $R 9 A P^{-/-} g g R 9 A P$ rods. These results are consistent with the idea that high activity of the R9AP - RGS9-1 - G $\beta 5$ complex can be supported regardless of the means of membrane attachment (lipidation or a transmembrane domain) and that overexpression of ggR9AP allows faster than normal transducin deactivation.

\section{Discussion}

In this study, we demonstrated that the stabilization of RGS9$1 \cdot \mathrm{G} \beta 5$ in rods occurs through a simple mechanism of recruitment to cellular membranes. While this is normally achieved through binding to the membrane anchor protein R9AP, direct membrane attachment of RGS9-1 itself is also sufficient, and it does not matter whether membrane binding takes place within the outer segment or elsewhere in the cell. The N-terminal DEP domain of RGS9-1 has been demonstrated to destabilize the protein (Martemyanov et al., 2003; Anderson et al., 2007a), but even though R9AP and the related R7BP have both been shown to bind to the DEP domain, this physical interaction is not sufficient to protect RGS9-1 if the anchor proteins have lost their capacity to associate with membranes. It is clear that the particular means of membrane attachment does not define the ability of an anchor protein to protect RGS9-1: palmitoylated R7BP, transmembrane wild-type R9AP, and an artificially geranylgeranylated R9AP mutant were all capable of performing the protective function.

Why does membrane attachment rescue RGS9-1 from proteolysis? The N terminus of RGS9-1 contains six destabilizing KFERQ-like motifs (Anderson et al., 2007a), short sequences consisting of glutamine next to a tetrapeptide containing a combination of acidic, basic, and hydrophobic residues (Dice, 1990). These sequences facilitate binding to the heat shock protein Hsc70, which targets proteins for lysosomal degradation in a process known as chaperone-mediated autophagy (for review, see Orenstein and Cuervo, 2010). The role of chaperone-mediated autophagy in RGS9 proteolysis is further supported by the observation that inhibition of lysosomal cysteine proteases, but not inhibition of the proteasome, decreased the rate of RGS9-2 deg- 
radation in mouse brain slices (Anderson et al., 2007a). It would appear likely, then, that binding of RGS9-1 to soluble R9AP or R7BP does not sufficiently mask the KFERQ-like motifs to prevent shuttling to the lysosome. However, the stability of RGS9-1 when associated with membranes would seem to suggest either that RGS9-1 is completely hidden from Hsc70 at this location or that Hsc70 fails to physically extract it from the membrane.

It is notable that the RGS9-1 constructs anchored to membranes by either a transmembrane domain or acylation exhibited a strikingly different localization from what is normally observed for RGS9-1. Although successfully protected from proteolysis, the fusion constructs were unable to traffic to the rod outer segment, the compartment where the transducin GTPase-activating complex functions. This observation, in conjunction with the finding that RGS9-1 mislocalizes in R7BP-expressing rods (Cao et al., 2010), illustrates that protection of RGS9-1 can be made distinct from its delivery to the outer segment. By normally using R9AP to perform both functions, the rod cell is able to ensure that the vast majority of RGS9-1 $-\mathrm{G} \beta 5$ protected from proteolysis ultimately ends up in the outer segment, where it can be put to productive use. Because both membrane-anchored RGS9-1 constructs were absent from the outer segments of $R 9 A P^{-/-}$rods, it is highly probable that a targeting signal specifying exclusion from outer segments resides within RGS9-1 - G $\beta 5$ itself and is neutralized upon R9AP binding. An interesting property of R9AP is that its outer segment localization may not require a specific targeting sequence. Our exhaustive search for such a sequence in R9AP expressed in transgenic Xenopus yielded no positive identification of a targeting signal, instead suggesting that R9AP is delivered to the outer segment by passively following the massive flux of post-Golgi vesicular traffic directed to this organelle (Baker et al., 2008). The existence of intracellular targeting information encoded within an R7 - G $\beta 5$ protein complex may suggest a whole new layer of spatial regulation by which multiprotein signaling complexes are delivered to their sites of action in neurons. This is particularly intriguing because $\mathrm{R} 7 \cdot \mathrm{G} \beta 5$ proteins are able to associate with membrane proteins other than conventional anchors, most notably with G-protein-coupled receptors (Garzón et al., 2005; Psifogeorgou et al., 2007; Sandiford and Slepak, 2009) and components of the synaptic fusion complex (Charlton et al., 2008).

In the final set of experiments, we took advantage of the $R 9 A P^{-1-}$ ggR9AP transgenic mice to assess the physiological consequences of changing the means of membrane attachment of the GTPase-activating complex from a transmembrane domain to lipidation. Our most straightforward finding is that this complex remained catalytically active and its overexpression resulted in response recovery rates similar to that observed when the complex anchored by normal R9AP is overexpressed. In fact, one might have expected the cells expressing high amounts of ggR9AP to recover even faster since peripheral membrane proteins diffuse much faster than transmembrane proteins, as established in many studies of isoprenylated proteins (Niv et al., 1999; Rotblat et al., 2004). As a rule, their diffusion coefficients are in the same range as membrane lipids, $\sim 1-2 \mu \mathrm{m}^{2} / \mathrm{s}$, whereas the diffusion coefficients of transmembrane proteins are at least 10 times lower and vary from 0.001 to $0.1 \mu \mathrm{m}^{2} / \mathrm{s}$ (for a comprehensive review, see Henis, 1993). Accordingly, the diffusion rate of RGS9-1 - G $\beta 5$ bound to ggR9AP may be predicted to be $>10$ fold faster than that of RGS9-1 - G $\beta 5$ bound to wild-type R9AP. Yet the fast subpopulations of rods from each ggR9AP line did not surpass the inactivation rate observed in conventional R9AP overexpressors. Three possible explanations described below could be considered.

First, it might be that ggR9AP - RGS9-1 - G $\beta 5$ expression levels within the individual rods recorded never reached the fourfold overexpression achieved in conventional R9AP overexpressors, such that, even with a higher diffusion rate, the lipid-anchored complex failed to encounter $\mathrm{G} \alpha_{\mathrm{t}} \cdot \mathrm{GTP}$ any more rapidly than the complex slowed by a transmembrane domain. However, this would seem unlikely, given that one of our transgenic mouse lines (line 4) exhibited a 4.9-fold overexpression of RGS9-1, despite electrophysiological evidence that roughly one-third of its rods did not express the ggR9AP transgene at all. Second, it could be that transducin inactivation had been accelerated to the point that its rate was now limited by the actual catalysis of GTP hydrolysis on $\mathrm{G} \alpha_{\mathrm{t}}$ rather than by the encounter rate between $\mathrm{G} \alpha_{\mathrm{t}} \cdot \mathrm{GTP}$ and the GTPase-activating complex. Third, it could be that ggR9AP did indeed allow for more rapid transducin inactivation in vivo when compared with the normal R9AP overexpressors but that this effect was masked by some other process that had become rate-limiting in these rods.

In summary, our finding that a mechanism as simple as membrane recruitment is sufficient for intracellular protection of a highly labile R7 RGS protein in photoreceptors implies that maintenance of its physiological level is not constrained by any specific protein-protein interaction. This result is particularly exciting in the context of the entire R7 family, the members of which engage in a rapidly expanding network of interactions with both conventional anchors and a diverse array of other membrane proteins.

\section{References}

Anderson GR, Semenov A, Song JH, Martemyanov KA (2007a) The membrane anchor R7BP controls the proteolytic stability of the striatal specific RGS protein, RGS9-2. J Biol Chem 282:4772-4781.

Anderson GR, Lujan R, Semenov A, Pravetoni M, Posokhova EN, Song JH, Uversky V, Chen CK, Wickman K, Martemyanov KA (2007b) Expression and localization of RGS9-2/G 5/R7BP complex in vivo is set by dynamic control of its constitutive degradation by cellular cysteine proteases. J Neurosci 27:14117-14127.

Anderson GR, Posokhova E, Martemyanov KA (2009) The R7 RGS protein family: multi-subunit regulators of neuronal $G$ protein signaling. Cell Biochem Biophys 54:33-46.

Baker SA, Martemyanov KA, Shavkunov AS, Arshavsky VY (2006) Kinetic mechanism of RGS9-1 potentiation by R9AP. Biochemistry 45:10690-10697.

Baker SA, Haeri M, Yoo P, Gospe SM 3rd, Skiba NP, Knox BE, Arshavsky VY (2008) The outer segment serves as a default destination for the trafficking of membrane proteins in photoreceptors. J Cell Biol 183:485-498.

Bownds D, Gordon-Walker A, Gaide-Huguenin AC, Robinson W (1971) Characterization and analysis of frog photoreceptor membranes. J Gen Physiol 58:225-237.

Cao Y, Kolesnikov AV, Masuho I, Kefalov VJ, Martemyanov KA (2010) Membrane anchoring subunits specify selective regulation of RGS9.G $\beta 5$ GAP complex in photoreceptor neurons. J Neurosci 30:13784-13793.

Charlton JJ, Allen PB, Psifogeorgou K, Chakravarty S, Gomes I, Neve RL, Devi LA, Greengard P, Nestler EJ, Zachariou V (2008) Multiple actions of spinophilin regulate mu opioid receptor function. Neuron 58:238-247.

Chen CK, Burns ME, He W, Wensel TG, Baylor DA, Simon MI (2000) Slowed recovery of rod photoresponse in mice lacking the GTPase accelerating protein RGS9-1. Nature 403:557-560.

Chen CK, Eversole-Cire P, Zhang H, Mancino V, Chen YJ, He W, Wensel TG, Simon MI (2003) Instability of GGL domain-containing RGS proteins in mice lacking the $\mathrm{G}$ protein beta-subunit Gbeta5. Proc Natl Acad Sci U S A 100:6604-6609.

Dice JF (1990) Peptide sequences that target cytosolic proteins for lysosomal proteolysis. Trends Biochem Sci 15:305-309.

Drenan RM, Doupnik CA, Boyle MP, Muglia LJ, Huettner JE, Linder ME, Blumer KJ (2005) Palmitoylation regulates plasma membrane-nuclear 
shuttling of R7BP, a novel membrane anchor for the RGS7 family. J Cell Biol 169:623-633.

Drenan RM, Doupnik CA, Jayaraman M, Buchwalter AL, Kaltenbronn KM, Huettner JE, Linder ME, Blumer KJ (2006) R7BP augments the function of $\mathrm{RGS}^{\star}{ }^{\mathrm{Gbeta} 5}$ complexes by a plasma membrane-targeting mechanism. J Biol Chem 281:28222-28231.

Garzón J, Rodríguez-Muñoz M, López-Fando A, Sánchez-Blázquez P (2005) Activation of mu-opioid receptors transfers control of Galpha subunits to the regulator of G-protein signaling RGS9-2: role in receptor desensitization. J Biol Chem 280:8951-8960.

Gray PC, Greenwald J, Blount AL, Kunitake KS, Donaldson CJ, Choe S, Vale W (2000) Identification of a binding site on the type II activin receptor for activin and inhibin. J Biol Chem 275:3206-3212.

Gross OP, Burns ME (2010) Control of rhodopsin's active lifetime by arrestin-1 expression in mammalian rods. J Neurosci 30:3450-3457.

Henis YI (1993) Lateral and rotational diffusion in biological membranes. In: Biomembranes: biophysical aspects (Shinitzki M, ed), pp 279-340. Weinheim, Germany; New York: VCH Publishers.

Hu G, Wensel TG (2002) R9AP, a membrane anchor for the photoreceptor GTPase accelerating protein, RGS9-1. Proc Natl Acad Sci U S A 99:9755-9760.

Hu G, Zhang Z, Wensel TG (2003) Activation of RGS9-1GTPase acceleration by its membrane anchor, R9AP. J Biol Chem 278:14550-14554.

Jayaraman M, Zhou H, Jia L, Cain MD, Blumer KJ (2009) R9AP and R7BP: traffic cops for the RGS7 family in phototransduction and neuronal GPCR signaling. Trends Pharmacol Sci 30:17-24.

Keresztes G, Mutai H, Hibino H, Hudspeth AJ, Heller S (2003) Expression patterns of the RGS9-1 anchoring protein R9AP in the chicken and mouse suggest multiple roles in the nervous system. Mol Cell Neurosci 24:687-695.

Keresztes G, Martemyanov KA, Krispel CM, Mutai H, Yoo PJ, Maison SF, Burns ME, Arshavsky VY, Heller S (2004) Absence of the RGS9.Gbeta5 GTPase-activating complex in photoreceptors of the R9AP knockout mouse. J Biol Chem 279:1581-1584.

Krispel CM, Chen CK, Simon MI, Burns ME (2003) Prolonged photoresponses and defective adaptation in rods of $\mathrm{G} \beta 5^{-1-}$ mice. J Neurosci 23:6965-6971.

Krispel CM, Chen D, Melling N, Chen YJ, Martemyanov KA, Quillinan N, Arshavsky VY, Wensel TG, Chen CK, Burns ME (2006) RGS expression rate-limits recovery of rod photoresponses. Neuron 51:409-416.

Lem J, Applebury ML, Falk JD, Flannery JG, Simon MI (1991) Tissuespecific and developmental regulation of rod opsin chimeric genes in transgenic mice. Neuron 6:201-210.

Lishko PV, Martemyanov KA, Hopp JA, Arshavsky VY (2002) Specific binding of RGS9-Gbeta 5L to protein anchor in photoreceptor membranes greatly enhances its catalytic activity. J Biol Chem 277:24376-24381.

Makino ER, Handy JW, Li T, Arshavsky VY (1999) The GTPase activating factor for transducin in rod photoreceptors is the complex between RGS9 and type 5 G protein beta subunit. Proc Natl Acad Sci U S A 96:1947-1952.

Martemyanov KA, Arshavsky VY (2009) Biology and functions of the RGS9 isoforms. Prog Mol Biol Transl Sci 86:205-227.

Martemyanov KA, Lishko PV, Calero N, Keresztes G, Sokolov M, Strissel KJ, Leskov IB, Hopp JA, Kolesnikov AV, Chen CK, Lem J, Heller S, Burns ME, Arshavsky VY (2003) The DEP domain determines sub- cellular targeting of the GTPase activating protein RGS9 in vivo. J Neurosci 23:10175-10181.

Martemyanov KA, Yoo PJ, Skiba NP, Arshavsky VY (2005) R7BP, a novel neuronal protein interacting with RGS proteins of the R7 family. J Biol Chem 280:5133-5136.

Martemyanov KA, Krispel CM, Lishko PV, Burns ME, Arshavsky VY (2008) Functional comparison of RGS9 splice isoforms in a living cell. Proc Natl Acad Sci U S A 105:20988-20993.

Masuho I, Wakasugi-Masuho H, Posokhova EN, Patton JR, Martemyanov KA (2011) Type $5 \mathrm{G}$ protein $\beta$ subunit (G $\beta 5$ ) controls the interaction of regulator of G protein signaling 9 (RGS9) with membrane anchors. J Biol Chem 286:21806-21813.

Matsuda T, Cepko CL (2004) Electroporation and RNA interference in the rodent retina in vivo and in vitro. Proc Natl Acad Sci U S A 101:16-22.

McCabe JB, Berthiaume LG (1999) Functional roles for fatty acylated amino-terminal domains in subcellular localization. Mol Biol Cell 10:3771-3786.

Niv H, Gutman O, Henis YI, Kloog Y (1999) Membrane interactions of a constitutively active GFP-Ki-Ras $4 \mathrm{~B}$ and their role in signaling. Evidence from lateral mobility studies. J Biol Chem 274:1606-1613.

Orenstein SJ, Cuervo AM (2010) Chaperone-mediated autophagy: molecular mechanisms and physiological relevance. Semin Cell Dev Biol 21:719-726.

Pepperberg DR, Cornwall MC, Kahlert M, Hofmann KP, Jin J, Jones GJ, Ripps H (1992) Light-dependent delay in the falling phase of the retinal rod photoresponse. Vis Neurosci 8:9-18.

Porter MY, Xie K, Pozharski E, Koelle MR, Martemyanov KA (2010) A conserved protein interaction interface on the type $5 \mathrm{G}$ protein beta subunit controls proteolytic stability and activity of R7 family regulator of $\mathrm{G}$ protein signaling proteins. J Biol Chem 285:41100-41112.

Psifogeorgou K, Papakosta P, Russo SJ, Neve RL, Kardassis D, Gold SJ, Zachariou V (2007) RGS9-2 is a negative modulator of mu-opioid receptor function. J Neurochem 103:617-625.

Pugh EN Jr (2006) RGS expression level precisely regulates the duration of rod photoresponses. Neuron 51:391-393.

Ross EM, Wilkie TM (2000) GTPase-activating proteins for heterotrimeric G proteins: regulators of $\mathrm{G}$ protein signaling (RGS) and RGS-like proteins. Annu Rev Biochem 69:795-827.

Rotblat B, Prior IA, Muncke C, Parton RG, Kloog Y, Henis YI, Hancock JF (2004) Three separable domains regulate GTP-dependent association of H-ras with the plasma membrane. Mol Cell Biol 24:6799-6810.

Sandiford SL, Slepak VZ (2009) The Gbeta5-RGS7 complex selectively inhibits muscarinic M3 receptor signaling via the interaction between the third intracellular loop of the receptor and the DEP domain of RGS7. Biochemistry 48:2282-2289.

Sokolov M, Strissel KJ, Leskov IB, Michaud NA, Govardovskii VI, Arshavsky VY (2004) Phosducin facilitates light-driven transducin translocation in rod photoreceptors. Evidence from the phosducin knockout mouse. J Biol Chem 279:19149-19156.

Watson AJ, Aragay AM, Slepak VZ, Simon MI (1996) A novel form of the G protein beta subunit Gbeta5 is specifically expressed in the vertebrate retina. J Biol Chem 271:28154-28160.

Wensel TG (2008) Signal transducing membrane complexes of photoreceptor outer segments. Vision Res 48:2052-2061. 